\title{
Análisis de calidad de vida laboral y competitividad en empresas de servicios turísticos
}

\section{Quality of work life and competitiveness in tourist service company}

José Octavio Molina Germán

Profesor de tiempo completo, adscrito al Departamento de ciencias económico-administrativas y al programa educativo de Administración de empresas. Universidad de Occidente, Unidad Mazatlán. octavio.udo@hotmail.com

Anel Yadira Pérez Melo

Profesora de Tiempo Completo adscrita al Departamento de Ciencias Económico-Administrativas y al programa educativo de Administración Turística. Miembro del Cuerpo Académico Capital Humano en las Organizaciones. Universidad de Occidente, Unidad Mazatlán. anel.perez@udo.mx

Georgina Lizárraga Salazar

Profesora de Tiempo Completo adscrita al Departamento de Ciencias Sociales y Humanidades y al programa educativo de Psicología. Miembro del Cuerpo Académico Capital Humano en las

Organizaciones. Universidad de Occidente, Unidad Mazatlán. goguil2002@hotmail.com

Ana María Larrañaga Núñez

Profesora de Tiempo Completo adscrita al Departamento de Ciencias Sociales y Humanidades y al programa educativo de Comunicación. Miembro del Cuerpo Académico Capital Humano en las Organizaciones. Universidad de Occidente, Unidad Mazatlán. amlarranaga2607@gmail.com

Recepción: 17/10/2017. Aceptación: 24/01/2018. Publicación: 24/05/2018

Citación sugerida:

Molina Germán, J.O., et al. (2018). Análisis de calidad de vida laboral y competitividad en empresas de servicios turísticos. 3C Empresa: investigación y pensamiento crítico, 7(2), 44-67. DOI: http://dx.doi. org/10.17993/3cemp.2018.070234.44-67/ 


\section{RESUMEN}

El estudio tiene como propósito analizar la relación e influencia de la calidad de vida laboral en el constructo de la competitividad en las empresas de servicios turísticos, particularmente en la hotelería. Para ello, se utilizó una metodología cuantitativa aplicando un cuestionario para contrastar la percepción de las dos variables dirigido a personal directivo y operativo de una empresa de hospedaje de la ciudad de Mazatlán, Sinaloa, México, mismos que fueron seleccionados de forma aleatoria. Los resultados obtenidos muestran similitudes en la percepción del personal sobre la calidad de vida laboral y la competitividad. No obstante, los datos reflejan una relación entre ambas variables casi nula en la percepción de los directivos y medianamente significativa desde la perspectiva de los operativos.

\section{ABSTRACT}

The purpose of the study is analyze the relationship and influence of the quality of working life on the competitiveness in tourism services companies, particularly in the hotel industry. A quantitative methodology was used applying a questionnaire to contrast the perception of the two variables directed to management and operating personnel of a hotel in the city of Mazatlan, Sinaloa, Mexico, which were selected randomly. The results show similarities in the staffs perception of the quality of work life and competitiveness. However, the data show a relationship between the two variables almost null in the manager's perception and medium-significant from employees' perspective.

\section{PALABRAS CLAVE}

Calidad de vida laboral, Competitividad, Empresa de hospedaje, Dimensiones.

\section{KEY WORDS}

Quality working life, Competitiveness, Tourism service companies, Dimensions. 


\section{INTRODUCCIÓN}

Las trasformaciones en el entorno mundial, así como las exigencias de los consumidores e inversionistas, representan tendencias que ejercen una constante presión para que las empresas generen ambientes de trabajo justos y empleados productivos, lo que evidencia la necesidad de reconfigurar los ambientes laborales en aras de garantizar un servicio de alto nivel competitivo.

Zohurul y Siengthai (2009), establecen que uno de los recursos más valiosos para las organizaciones son sus trabajadores, quienes a través de su esfuerzo logran que las empresas perduren y obtengan mejores resultados organizacionales. Así, la productividad y los resultados de la empresa están influenciados por la Calidad de Vida Laboral (CVL) que experimentan sus trabajadores.

Los cambios significativos que han ocurrido en los últimos años han generado condiciones distintas en las perspectivas de los mercados turísticos mundiales, planteando nuevos retos y oportunidades para las empresas de este sector.

La productividad y los resultados de la empresa están influenciados por la Calidad de Vida Laboral (CVL) que experimentan sus trabajadores.

De acuerdo con la Secretaria de Turismo, la actividad turística en México representa el 9\% del Producto Interno Bruto (PIB) y da empleo a más de 7,5 millones de personas (SECTUR, 2014). En el estado de Sinaloa, durante el primer trimestre del 2014, se tuvo un porcentaje de ocupación del 48.2\% a nivel estatal y un 51.3\% en la ciudad de Mazatlán (SECTUR, 2014).

Esta dinámica transforma el quehacer de la gestión directiva y establece la necesidad de analizar y determinar cuáles son aquellos factores, programas y estrategias que harían posible transitar hacia la competitividad.

Al ser este último elemento de naturaleza multifactorial, se considera que un factor de alta significancia se refiere a la calidad de vida en el trabajo, por lo que su estudio se hace necesario e indispensable a fin de entender su complejidad. 


\section{ESTUDIOS PREVIOS}

El análisis de la calidad de vida laboral es de importancia creciente dadas las nuevas formas de organización empresarial y del trabajo que generan en los individuos altos niveles de presión y de estrés laboral, lo que impacta en el rendimiento y en consecuencia en los resultados productivos.

Se concibe, a partir de estas consideraciones, que el análisis de la calidad de vida laboral constituye un elemento básico y diferenciador que puede potenciarse como una muy valiosa ventaja competitiva. Podemos destacar algunos estudios realizados que revelan que la calidad de vida laboral influye sobre el resultado organizacional.

Lau y May, (1998) y Harter, Schmidt y Hayes, (2002), establecen que la Calidad de Vida Laboral (CVL) ofrecida por una organización y percibida como satisfactoria por los empleados, repercute positivamente en la salud física, psíquica y emocional del trabajador, impactando favorablemente en los desempeños y resultados organizacionales.

\section{El análisis de la calidad de vida laboral constituye un elemento básico y diferenciador que puede potenciarse como una muy valiosa ventaja competitiva.}

Asimismo, los estudios realizados por Efraty y Sirgy (1990), demuestran que las organizaciones que generan bienes y/o servicios de calidad a través de adecuadas condiciones de trabajo, así como oportunidades de desarrollo personal/profesional para sus empleados, también son las que obtienen mayores ganancias y mejor consideración social.

Por otra parte, Zohurul y Siengthai (2009), realizan un estudio exploratorio de la influencia de la CVL sobre la satisfacción laboral y el resultado organizacional. Sus descubrimientos indican que la CVL sugiere que la calidad de vida laboral está positivamente relacionada al resultado organizacional pero no fue significativa, sin embargo, encuentran una relación positiva y significativa entre la calidad de vida laboral y la satisfacción laboral de los empleados.

Asimismo, Sauter, Lim y Murphy (1996) reconocen que el resultado organizacional y el bienestar de los trabajadores se refuerzan mutuamente e introducen un modelo desarrollado por el Instituto Nacional de Seguridad Ocupacional y Salud (NIOSH) en su investigación de organizaciones de trabajo saludable. 
Para Sánchez (2013), la GVL se debe adaptar a las particularidades de la organización, considerando el propósito de competitividad de las organizaciones. Asimismo, es necesario compaginar la CVL desde de la organización y desde del trabajador, pues la organización no funciona sin talentos que la operen e impulsen su CVL, crecimiento y competitividad.

En lo que respecta a Argüelles, Quijano y Fajardo (2017) realizaron una investigación en empresas turísticas de Campeche sobre la relación e influencia de la CVL con los resultados de las organizaciones, encontraron que al establecer estrategias enfocadas a mejorar la CVL de los trabajadores esto se refleja en el rendimiento y beneficio de la organización a corto, mediano o largo plazo. 


\section{CALIDAD DE VIDA LABORAL}

El término Calidad de Vida Laboral (CVL), es de gran relevancia en el estudio del capital humano y ha sido abordado desde diferentes perspectivas como se visualiza en las investigaciones realizadas por múltiples autores como Kraut, Dumais y Koch, 1989; Pruijt, 2000; Joshi, Leichne, Melanson, Pruna, Sager, Jo Store, Williams, 2002; Shoaf, Genaidy, Karwowski y Huang, 2004; Cole, Robson, Lemieux-Charles, McGuire, Sicotte y Champagne, 2005; Hart, Ribbing, Abrahamsson, 2005; Che, Beh, Uli y Idris, 2006; Ukko, Tenhunen, y Rantanen, 2008; Zohurul y Siengthai, 2009, (citado por Contreras; Huerta; Pedraja \& Almodóvar Martínez, 2011).

Este concepto presenta cierta dificultad para definirse debido a las características de subjetividad y multidimensionalidad que posee. Es decir, depende de los valores y creencias de los individuos, de aspectos culturales, de historias de vida así como de la valoración que se dé a las necesidades humanas. Por ejemplo, para González (2009), la calidad de vida da cuenta del problema de satisfacción de necesidades, según French (1996), la calidad de vida en el trabajo (CVT) es una filosofía de gestión que mejora la dignidad del empleado, realiza cambios culturales y brinda oportunidades de desarrollo y progreso personal. Por su parte, Gibson (1996) agrega que es una filosofia, un conjunto de creencias que engloban los esfuerzos por incrementar la productividad y mejorar la motivación de las personas, enfatizando la participación de la gente, su dignificación y la eliminación de aspectos disfuncionales de la jerarquía organizacional.

Es necesario señalar que distintos autores hacen la observación de la dificultad que tiene este concepto para operacionalizarse, por lo que cada uno propone diversas variables de abordaje. Para Poza y Prior (1988, citado en Gamero, 2003, p.35) la CVL es la forma en que se produce la experiencia laboral en condiciones objetivas como seguridad e higiene laboral, trabajo, salud laboral, entre otros, así como las condiciones subjetivas del trabajador de cómo lo percibe o vive. Según Toro (2001), la Calidad de Vida Laboral se refiere a aspectos objetivos como son las condiciones y realidades del trabajo que buscan el bienestar y desarrollo de los empleados, considerando aspectos como la retribución, condiciones de salubridad, seguridad e higiene, ambiente social, liderazgo, trato equitativo, entre otros, incluyendo la percepción y la evaluación subjetiva que hace el individuo a las situaciones antes señaladas.

Por otro lado, Havlovic (1991) y Scobel (1975) señalan que algunos de los elementos clave de la CVL son seguridad en el trabajo, satisfacción laboral, sistemas de recompensas, involucramiento de los empleados y resultados organizacionales, (citado en Contreras et al, 2013). Ukko Tenhunen, y Rantanen (2008: 87) afirman que incluye ocho elementos: motivación en el trabajo, oportunidades 
de aprendizaje, satisfacción en el trabajo, atmósfera de trabajo, salud y seguridad, participación en la toma de decisiones, realización de objetivos a nivel personal y de equipo, y sistema de recompensa.

Una clasificación de GVL muy aplicada por investigadores es la que incorpora por un lado la perspectiva del entorno de trabajo, con indicadores centrados en los intereses organizacionales (condiciones y medio ambiente de trabajo, cultura y clima laboral, productividad y efectividad, condiciones de vida, entre otros), y por otro lado una perspectiva psicológica centrada en el individuo (actitudes y valores hacia el trabajo, motivación y satisfacción laboral), Chiang y Krausee, (2008).

Para el objeto de estudio, se seleccionaron las siguientes dimensiones por considerarlas de gran importancia para la medición y observación de la variable de calidad de vida laboral y su relación con la competitividad de la organización.

a) Desarrollo Profesional. Es el proceso por el que las personas progresan a través de una serie de etapas caracterizadas por distintas tareas de desarrollo, actividades y relaciones (Fernández, 2002, p.67).

b) Beneficios socioeconómicos. Incluye las políticas de remuneración, compensación e incentivos, de los trabajadores.

c) Satisfacción en el Trabajo. Abarca diferentes aspectos como el trabajo en sí, salarios e incentivos, supervisión, promociones, ambiente de trabajo, entre otros.

d) Sentido de Pertenencia. La capacidad del individuo de sentirse integrante de un grupo u organización. En la relación empresa-empleado, la pertenencia permite alcanzar objetivos individuales y organizacionales.

e) Participación del Personal. Contempla las capacidades que posee el personal y la apertura de la empresa para el uso de las mismas, así como su participación en la toma de decisiones.

f) Medio ambiente de Trabajo. Factores ambientales que pueden ocasionar enfermedades o efectos negativos en el bienestar del personal. Incluye asimismo la prevención de los accidentes y enfermedades.

Como se observa, la CVL es un proceso que permite a las organizaciones responder a las necesidades de sus empleados para mejorar su vida laboral, su desarrollo profesional y personal. Por lo tanto, se puede decir que la CVL, busca el desarrollo del trabajador, como medio para elevar la eficiencia empresarial. 


\section{COMPETITIVIDAD}

A partir de las exigencias del entorno económico actual y al proceso de globalización en las que se encuentran inmersas las organizaciones, un aspecto que adquiere mayor relevancia es la competitividad. Esto se debe principalmente porque las empresas están obligadas a mantener e incrementar su participación en el mercado, a innovar sus productos y servicios, a flexibilizar sus sistemas de producción, a tener una rápida respuesta a las demandas del entorno, todo ello con una reducción en sus costos y un aumento en su productividad.

No obstante, se considera que la competitividad es un concepto complejo, el cual puede ser estudiado desde diferentes enfoques y disciplinas, incluso algunos autores señalan que no es posible establecer una definición única, en parte debido a que su utilidad reside en identificar vías para fomentar empresas que contribuyan a elevar los niveles reales de bienestar (Solleiro y Castañón, 2005).

Se destacan dos grandes enfoques para abordar la competitividad, uno de ellos es el propuesto por World Competitiveness Center (WCG) del Institute for Management and Development, el cual indica que es el ambiente competitivo de las naciones el que crea y mantiene la competitividad de las empresas. El otro es del Institute for Strategy and Competitiveness (ISC) de la Universidad de Harvard, éste señala que las condiciones macroeconómicas dan las oportunidades pero son las empresas las que crean bienes y servicios con valor agregado usando métodos eficientes.

Bajo estas perspectivas, la competitividad está relacionada con la capacidad de incrementar el nivel de vida de los habitantes, de generar incrementos sostenidos en productividad, de insertarse exitosamente en los mercados internacionales, entre otros (Padilla, 2006, citado por Saavedra, 2012).

Sin embargo, autores como Porter (1990) han señalado que las que compiten son las empresas no las naciones, a un país lo hacen competitivo las empresas competitivas que hay en este. Por lo tanto, son estas la base de la competitividad.

La competitividad empresarial es la capacidad para, rivalizando con otras empresas, alcanzar una posición competitiva favorable, que permita obtener un desempeño superior al de los competidores (Rubio, 2004, Citado en Rubio y Aragón, 2006, p. 105). Solleiro y Castañón (2005), señalan que es la capacidad de una organización para mantener su participación en el mercado basada en nuevas estrategias empresariales, en el crecimiento sostenido de la productividad y en la capacidad para participar en negociaciones con diferentes instituciones. 
La Teoría de los Recursos y las Capacidades sontiene que la rentabilidad de una empresa se encuentra estrechamente vinculada con el tipo, cantidad y naturaleza de sus recursos y capacidades.

La escuela de los recursos internos (Wernerfelt, 1991; Amit y Schoemaker, 1993; Hoopes, Madsen \& Walker, 2003), sostiene que las empresas disponen de recursos y capacidades únicos, que les proporcionan la base para la estrategia.

Bajo este enfoque surge la teoría de los recursos y las capacidades (TRC). Esta sostiene que la rentabilidad de una empresa se encuentra estrechamente vinculada con el tipo, cantidad y naturaleza de sus recursos y capacidades. Amit y Schoemaker (1993), se refieren a estos componentes, como activos estratégicos, los cuales deben ser protegidos y desarrollados para lograr una ventaja competitiva. En esta idea, Prahalad y Hamel (1990) establecen que es un requisito fundamental para los altos ejecutivos poseer la capacidad de identificar, cultivar y aprovechar los recursos y capacidades de la empresa, que representen rentabilidad y crecimiento.

Bajo esta perspectiva se abordan como dimensiones de competitividad:

a) Recursos Tangibles. Elementos productivos físicos o medios financieros.

b) Recursos intangibles. Se clasifican en recursos humanos (formación, experiencia, adaptación), recursos tecnológicos (patentes, conocimiento, experiencia productiva) y reputación de la empresa frente a sus clientes y proveedores.

c) Capacidades. Habilidades y conocimientos que posee la organización para alcanzar objetivos deseados.

d) Competitividad empresarial. Dimensión de contraste para determinar la percepción del desarrollo de nuevos productos, satisfacción del cliente, interés por la formación y desarrollo del personal y por la imagen de la empresa. 


\section{METODOLOGÍA}

El estudio es descriptivo, transversal, correlacional, de corte cuantitativo donde se analiza las variables GVL y competitividad desde la percepción del personal directivo y operativo de una empresa de hospedaje de la ciudad de Mazatlán, Sinaloa.

La empresa fue seleccionada por su representatividad en la localidad, así como por las certificaciones que ostenta como empresa verde (certificación de Profepa), empresa incluyente, y Certificación Great Place To Work. En el momento de la aplicación se contaba con una población de 205 trabajadores, la muestra fue aleatoria integrada por 66 trabajadores que permitiría tener un margen de error de $10 \%$, sin embargo, se descartaron 8 instrumentos, quedando una muestra de 58 sujetos los cuales 10 tiene puesto directivos y el resto operativo. Algunos de los criterios que se consideraron fueron: a) incluir a trabajadores de las 3 jornadas laborales, b) que la muestra contemplara por lo menos $15 \%$ de directivos, c) contar con personal de todos los departamentos que conforman el hotel.

Para medir la GVL, se adaptó el cuestionario diseñado por Huerta, Soler y otros (2003) a partir de juicio de expertos (5) y prueba piloto, resultando un instrumento constituido por 53 ítems, relacionados con seis dimensiones e indicadores (ver tabla 1). 
Tabla 1. Dimensiones CVL.

\begin{tabular}{|c|c|}
\hline DIMENSIONES & INDICADORES \\
\hline Desarrollo profesional & $\begin{array}{l}\text { - Promoción } \\
\text { - Adiestramiento } \\
\text { - Planes de carrera }\end{array}$ \\
\hline Beneficios socioeconómicos & $\begin{array}{l}\text { - Salarios } \\
\text { - Bonos navideños } \\
\text { - Vacaciones } \\
\text { - Atención médica }\end{array}$ \\
\hline Satisfacción en el trabajo & $\begin{array}{l}\text { - Evaluación del desempeño Incentivo, Motivación, Clima } \\
\text { organizacional, Reconocimientos }\end{array}$ \\
\hline Sentido de pertenencia & $\begin{array}{l}\text { - Identidad con la tarea } \\
\text { - Identidad con la empresa } \\
\text { - Imagen corporativa }\end{array}$ \\
\hline Proceso de participación & $\begin{array}{l}\text { - Creatividad y expresión personal } \\
\text { - Repercusiones de ideas aportadas } \\
\text { - Programas de participación en la toma de decisiones }\end{array}$ \\
\hline Medio ambiente de trabajo & $\begin{array}{l}\text { - Higiene industrial } \\
\text { - Orden } \\
\text { - Seguridad } \\
\text { - lluminación } \\
\text { - Temperatura } \\
\text { - Ruidos } \\
\text { - Períodos de descanso }\end{array}$ \\
\hline
\end{tabular}

Fuente: elaboración propia (2017).

Para la variable competitividad, se utiliza el modelo planteado por Fonseca (2007), basado en la TRC. La dimensión recursos tangibles solo aplica al personal directivo. La versión para directivos cuenta con 50 ítems y para personal operativo 30 (ver tabla 2). 
Tabla 2. Dimensiones Competitividad.

\begin{tabular}{|c|c|}
\hline DIMENSIÓN & INDICADOR \\
\hline Recursos tangibles & $\begin{array}{l}\text { - Activo físico } \\
\text { - Activo financiero }\end{array}$ \\
\hline Recursos intangibles & $\begin{array}{l}\text { - Capital humano } \\
\text { - Recursos tecnológicos } \\
\text { - Reputación }\end{array}$ \\
\hline Capacidades & $\begin{array}{l}\text { Capacidad de marketing } \\
\text { Capacidad de distribución } \\
\text { Sistemas de información } \\
\text { Capacidad directiva }\end{array}$ \\
\hline Competitividad empresarial & $\begin{array}{l}\text { - Recursos } \\
\text { - Capacidades }\end{array}$ \\
\hline
\end{tabular}

Fuente: elaboración propia (2017).

El instrumento fue elaborado con escala tipo Likert de cinco alternativas de respuesta con rango $1=$ Muy en Desacuerdo a 5=Muy de Acuerdo.

Los resultados obtenidos se procesaron mediante el programa estadístico SPSS en su versión 22.0, realizando el análisis de confiabilidad, los resultados descriptivos, así como correlacionar las dimensiones de calidad de vida laboral con las variables de competitividad, los cuales se observan en el siguiente apartado. 


\section{RESULTADOS}

Los resultados de confiabilidad del alfa de cronbach arrojó 0,962 y 0,887 para CVL (directivos y operativos) y para competitividad 0,951 y 0,857 (directivos y operativos), por lo que el instrumento es confiable, tomando como referencia el criterio sugerido por George y Mallery (2003), quienes establecen las recomendaciones siguientes para evaluar los coeficientes: $>0.9$ excelente, $>0.8$ bueno y $>0.7$ aceptable.

En los datos sociodemográficos, respecto a directivos, el 40\% tiene antigüedad entre 6-10 años y el 30\% más de 10 años. Respecto a la edad, 40\% está en un rango de 31 a 35 años, un 40\% se divide de forma equitativa entre los 36 a 40 y los 41 a 45 años. El 90\% son hombres. El 90\% posee estudios de licenciatura y 10\% estudios de bachillerato; 60\% cuenta con más de 10 trabajadores a su cargo.

Respecto al personal operativo, el 37.5\% estudió hasta bachiller, 29.2\% secundaria, 14.6\% con licenciatura y $12.5 \%$ estudios comerciales. El 33.3\% tiene una antigüedad entre 3-5 años, 27.1\% menos de 3 años, 20.8\% más de 10 años y 18.8\% tiene entre 6-10 años. El 39.6\% son mujeres y $56.3 \%$ son hombres. Del total 29.2\% tiene una edad entre 18-25 años, 25\% se ubican entre 26-30 años, seguidos por el 20.8\% de 31-35 años igual que los de $41-45$ años, el 75\% está por debajo de los 36 años.

Tabla 3. Baremos y medias de Calidad de vida laboral global y sus dimensiones.

\begin{tabular}{|c|c|c|c|c|c|c|c|c|}
\hline & CVL GLOBAL & $\begin{array}{c}\text { DESARROLLO } \\
\text { PROFESIONAL }\end{array}$ & $\begin{array}{c}\text { BENEFICIOS } \\
\text { SOCIOECONÓMICOS }\end{array}$ & $\begin{array}{c}\text { SATISFACCIÓN } \\
\text { EN EL TRABAJO }\end{array}$ & $\begin{array}{c}\text { SENTIDO DE } \\
\text { PERTENENCIA }\end{array}$ & $\begin{array}{c}\text { PROCESO DE } \\
\text { PARTICIPACIÓN }\end{array}$ & $\begin{array}{c}\text { MEDIO AMBIENTE } \\
\text { DE TRABAJO }\end{array}$ \\
\hline & RANGOS & RANGOS & RANGOS & RANGOS & RANGOS & RANGOS & RANGOS \\
\hline CRITERIOS & DIR OPER & DIR OPER & DIR OPER & DIR OPER & DIR OPER & DIR & OPER & DIR OPER \\
\hline BAJO & $0-123$ & $0-16$ & $0-16$ & $0-22$ & $0-16$ & $0-23$ & $0-28$ \\
\hline MEDIO & $124-194$ & $17-25$ & $17-25$ & $23-36$ & $17-25$ & $24-36$ & $29-44$ \\
\hline ALTO & $195-265$ & $26-35$ & $26-35$ & $37-50$ & $26-35$ & $37-50$ & $45-60$ \\
\hline MEDIA & $\mathbf{2 3 8 - 2 1 7}$ & $\mathbf{3 2}-\mathbf{3 0}$ & $\mathbf{3 2}-\mathbf{2 9}$ & $\mathbf{4 5}-\mathbf{4 2}$ & $\mathbf{3 2}-\mathbf{3 0}$ & $\mathbf{4 4}-\mathbf{3 6}$ & $\mathbf{5 3}-\mathbf{5 0}$ \\
\hline
\end{tabular}

Fuente: elaboración propia (2017). 


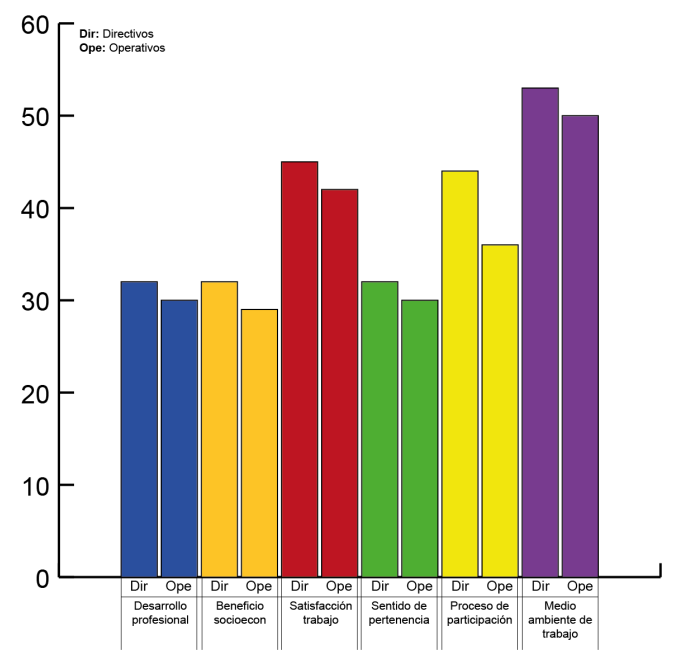

Gráfica 1. Medias de las dimensiones de Calidad de vida laboral. Fuente: elaboración propia (2017).

Los resultados para la dimensión global de calidad de vida laboral en la tabla 3, muestran una media de 237.75 para los directivos y 216.90 para el personal operativo, lo que ubica a esta variable en un resultado alto de acuerdo con los criterios establecidos en los baremos.

Los resultados de las dimensiones desarrollo profesional, beneficios socioeconómicos, satisfacción en el trabajo, sentido de pertenencia, procesos de participación y medio ambiente de trabajo, (tabla 3 y gráfica 1), muestran una tendencia favorable, ya que las medias que se obtienen para directivos y operativos reflejan una puntuación alta. 
Tabla 4. Baremos y medias de Competitividad y sus dimensiones.

\begin{tabular}{|c|c|c|c|c|c|c|c|c|c|}
\hline \multirow[b]{3}{*}{ CRITERIOS } & \multirow{2}{*}{\multicolumn{2}{|c|}{$\begin{array}{c}\text { COMPETITIVIDAD } \\
\text { GLOBAL } \\
\text { RANGOS }\end{array}$}} & \multirow{3}{*}{$\begin{array}{l}\text { RECURSOS } \\
\text { TANGIBLES* } \\
\text { RANGOS } \\
\text { DIR }\end{array}$} & \multirow{2}{*}{\multicolumn{2}{|c|}{$\begin{array}{l}\text { RECURSOS } \\
\text { INTANGIBLES } \\
\text { RANGOS }\end{array}$}} & \multirow{2}{*}{\multicolumn{2}{|c|}{$\begin{array}{l}\text { CAPACIDADES } \\
\text { RANGOS }\end{array}$}} & \multirow{2}{*}{\multicolumn{2}{|c|}{$\begin{array}{c}\text { COMPETITIVIDAD } \\
\text { EMPRESARIAL } \\
\text { RANGOS }\end{array}$}} \\
\hline & & & & & & & & & \\
\hline & DIR & OPER & & DIR & OPER & DIR & OPER & DIR & OPER \\
\hline BAJO & $0-177$ & $0-70$ & $0-21$ & $0-42$ & $0-35$ & $0-33$ & $0-16$ & $0-21$ & $0-14$ \\
\hline MEDIO & $118-185$ & $71-110$ & $22-33$ & $43-66$ & $36-55$ & $34-52$ & $17-25$ & $22-33$ & $15-22$ \\
\hline ALTO & $186-250$ & $111-150$ & $34-45$ & $67-90$ & $56-75$ & $53-70$ & $26-35$ & $34-45$ & $23-30$ \\
\hline MEDIA & 217 & 127 & 38 & 78 & 67 & 60 & 29 & 40 & 25 \\
\hline
\end{tabular}

Fuente: elaboración propia (2017).

* Solo aplica para directivos.

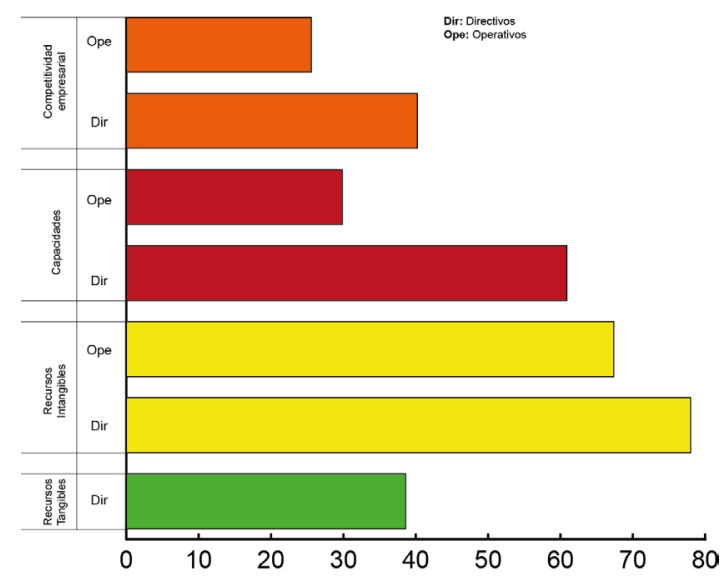

Gráfica 2. Medias de las dimensiones de Competitividad.

Fuente: elaboración propia (2017) 
Tabla 5. Correlación entre las variables Calidad de vida laboral y competitividad en Directivos.

\begin{tabular}{|c|c|c|c|c|c|}
\hline 1. Recursos tangibles & & \multirow{4}{*}{1} & \multirow{4}{*}{2} & \multirow{4}{*}{3} & \multirow{4}{*}{4} \\
\hline 2. Recursos intangibles & & & & & \\
\hline 3. Capacidades & & & & & \\
\hline 4. Competitividad empresarial & & & & & \\
\hline \multirow{2}{*}{ Desarrollo Profesional } & \multirow{2}{*}{ Correlación de Pearson Sig. } & .261 & .479 & .522 & .367 \\
\hline & & .467 & .161 & .150 & .332 \\
\hline \multirow{2}{*}{ Beneficios Socioeconómicos } & \multirow{2}{*}{ Correlación de Pearson Sig. } & -.058 & .188 & .294 & .480 \\
\hline & & .873 & .602 & .443 & .191 \\
\hline \multirow{2}{*}{ Satisfacción en el trabajo } & \multirow{2}{*}{ Correlación de Pearson Sig. } & .178 & .168 & -.006 & .496 \\
\hline & & .647 & .665 & .989 & .211 \\
\hline \multirow{2}{*}{ Sentido de pertenencia } & \multirow{2}{*}{ Correlación de Pearson Sig. } & .260 & .343 & .255 & .584 \\
\hline & & .468 & .332 & .508 & .098 \\
\hline \multirow{2}{*}{ Proceso de participación } & \multirow{2}{*}{ Correlación de Pearson Sig. } & .289 & .649 & .427 & $.830^{*}$ \\
\hline & & .450 & .059 & .292 & .011 \\
\hline \multirow{2}{*}{ Medio ambiente de trabajo } & \multirow{2}{*}{ Correlación de Pearson Sig. } & .341 & .367 & .328 & .430 \\
\hline & & .336 & .296 & .390 & .248 \\
\hline
\end{tabular}

La tabla anterior evidencia desde la perspectiva de análisis de la información que se obtuvo de los directivos, que no existe correlación entre las dimensiones de las variables calidad de vida laboral y competitividad, exceptuando la dimensión proceso de participación con competitividad empresarial que manifiesta una correlación 0,830 y una significancia para el nivel de confianza de 0,011.

Tabla 6. Correlación entre las variables Calidad de vida laboral y competitividad del personal operativo.

\begin{tabular}{|c|c|c|c|c|}
\hline 1. Recursos tangibles & & \multirow{4}{*}{1} & \multirow{4}{*}{2} & \multirow{4}{*}{3} \\
\hline 2. Recursos intangibles & & & & \\
\hline 3. Capacidades & & & & \\
\hline 4. Competitividad empresarial & & & & \\
\hline \multirow{2}{*}{ Desarrollo Profesional } & \multirow{2}{*}{ Correlación de Pearson Sig. } & .222 & .106 & .230 \\
\hline & & .142 & .485 & .124 \\
\hline \multirow{2}{*}{ Beneficios Socioeconómicos } & \multirow{2}{*}{ Correlación de Pearson Sig. } & -.239 & .179 & $.294^{*}$ \\
\hline & & .105 & .224 & .042 \\
\hline \multirow{2}{*}{ Satisfacción en el trabajo } & \multirow{2}{*}{ Correlación de Pearson Sig. } & $.297^{\star}$ & $.302^{*}$ & $.509^{\star *}$ \\
\hline & & .042 & .037 & .000 \\
\hline \multirow{2}{*}{ Sentido de pertenencia } & \multirow{2}{*}{ Correlación de Pearson Sig. } & $.446^{* *}$ & $.352^{*}$ & $.406^{* *}$ \\
\hline & & .002 & .015 & .005 \\
\hline \multirow{2}{*}{ Proceso de participación } & \multirow{2}{*}{ Correlación de Pearson Sig. } & .248 & .177 & .130 \\
\hline & & .101 & .239 & .388 \\
\hline \multirow{2}{*}{ Medio ambiente de trabajo } & \multirow{2}{*}{ Correlación de Pearson Sig. } & $.482^{* *}$ & $.302^{*}$ & .211 \\
\hline & & .001 & .039 & .155 \\
\hline
\end{tabular}


Desde la perspectiva del personal operativo, en la tabla 6 se muestra que existen relaciones moderadas entre la dimensión de satisfacción en el trabajo con la competitividad empresarial con un resultado de 0,509. Entre la dimensión de sentido de pertenencia respecto a los recursos intangibles, capacidades y la competitividad empresarial cuyos resultados son 0,446, 0,352 y 0,406 con valores de significancia de 0,002, 0,015 y 0,005. Entre la dimensión medio ambiente de trabajo respecto a los recursos intangibles y las capacidades que muestran una relación de 0,482 y 0,302 con valores de significancia de 0,001 y 0,039 respectivamente.

Se infiere que un porcentaje superior al 90\% del personal de la empresa estudiada considera que su CVL se encuentra a un nivel alto. Esto supone que la organización desarrolla actividades orientadas a la transformación de ideas y actitudes, que la remuneración y las políticas salariales son favorables, que diseña estrategias para la satisfacción personal y al mejoramiento de las condiciones de trabajo. Asimismo, que promueve una participación de su personal identificado con la tarea, empresa e imagen corporativa, incidiendo en el rendimiento y la competitividad de la organización. 


\section{DISCUSIÓN}

Con los resultados obtenidos, se puede inferir que un porcentaje superior al $90 \%$ del personal que labora en la empresa objeto de estudio, considera que su calidad de vida laboral se encuentra a un nivel alto. Esto indica que la empresa ofrece condiciones relacionadas con las dimensiones de calidad de vida laboral que genera una percepción de satisfacción y que ello se traduce en resultados favorables que impactan el rendimiento y la competitividad de la organización.

Los resultados en cuanto a la GVL, difieren con los obtenidos por Huerta, Soler y otros (2003), que presentan resultados ubicados en un nivel medio. Los datos que arroja esta investigación son mayormente puntuaciones favorables percibiendo a esta variable en el rango más alto de acuerdo con los criterios establecidos.

En lo que respecta a competitividad, se observa una percepción favorable por parte de directivos y personal operativo, ellos consideran que la organización logra sus objetivos y metas a través del uso adecuado de los recursos y desarrollo de estrategias eficaces.

Zohurul y Siengthai (2009), descubrieron que la GVL está relacionada al resultado organizacional pero no significativamente, excepto en la dimensión satisfacción laboral de los empleados, respecto a ello, encontramos similitudes principalmente en los datos de directivos. El estudio refleja una relación positiva y significativa entre la dimensión proceso de participación y la dimensión competitividad empresarial. En tanto los datos correspondientes al personal operativo evidencia relaciones moderadas en las dimensiones de satisfacción en el trabajo, sentido de pertenencia y medio ambiente de trabajo, respecto a la competitividad empresarial y los recursos intangibles. 


\section{CONCLUSIONES}

El estudio se realizó con el propósito de analizar la calidad de vida laboral y la competitividad percibida por los empleados de una organización turística con sede en la ciudad de Mazatlán, Sinaloa, México. Una vez procesada y analizada la información recabada mediante el trabajo de campo se puede establecer en términos generales que el nivel de calidad de vida laboral de los empleados en la empresa estudiada es alto, lo que indica que las personas perciben que la organización logra satisfacer de manera significativa las necesidades relacionadas con el desarrollo profesional, beneficios socioeconómicos, satisfacción en el trabajo, sentido de pertenencia y medio ambiente de trabajo.

Sólo la dimensión de proceso de participación para el personal operativo se ubicó en el límite superior del rango medio de la puntuación, entendiéndose este resultado como parte de un fenómeno generalizado que caracteriza a las organizaciones ya que en la mayoría de ellas los procesos de participación y la toma de decisiones representan acciones que regularmente se reservan para los cuadros o equipos de dirección.

\section{Las personas perciben que la organización logra satisfacer de manera significativa las necesidades relacionadas con el desarrollo profesional, beneficios socioeconómicos, satisfacción en el trabajo, sentido de pertenencia y medio ambiente de trabajo.}

En cuanto a los resultados de la variable competitividad, se observa una percepción favorable con rango más alto en las dimensiones de recursos tangibles, intangibles, capacidades y competitividad empresarial. Esto indica que la empresa emplea los recursos disponibles adecuadamente, desarrollando estrategias directivas eficaces orientadas a las actividades financieras, procesos de producción e innovación, investigación de mercados, mejoramiento de la calidad e imagen de la empresa, procesos de comunicación y participación, así como al desarrollo de las capacidades de los empleados.

Para futuros estudios es importante considerar el carácter multidimensional de la variable CVL y analizarla en contraste con otras variables para la comprensión multifactorial de esta variable. Asimismo, desarrollar estudios que permitan enfatizar en la correlación entre ambas dimensiones para definir a que factores puede ser posible atribuir una mayor influencia y con ello diseñar 
estrategias de mejoramiento de la competitividad de las empresas, asimismo, ampliar el estudio a otras empresas del sector para evaluar si existen diferencias significativas en los resultados. 


\section{REFERENCIAS BIBLIOGRÁFICAS}

Amit, R. y Schoemaker, P. (1993). Strategic assets and organizational rent. Strategic Management Journal, 14, 33-46.

Argüelles, M.A., Quijano, L.A. y Fajardo, M.A. (2017). Rentabilidad y Calidad de Vida Laboral, Revista Internacional Administración \& Finanzas, 10(1), pp. 81-93.

Chiang M., y Krausse A. (2008). Estudio empírico de calidad de vida laboral, cuatro indicadores: satisfacción laboral, condiciones y medioambiente del trabajo, organización e indicador global, sectores privado y público. Desarrollo, aplicación y validación del instrumento. Horizontes empresariales, 3, 23-50.

GODESIN. (2015). Reporte de llegada de turistas y ocupación hotelera en Sinaloa, de enero a diciembre de 2015. Disponible en: http://sinaloaennumeros.com/reporte-de-llegada-deturistas-y-ocupacion-hotelera-en-sinaloa-de-enero-a-diciembre-de-2015/

Efraty, D. y Sirgy, M. (1990). The effects of Quality of Working life (QWL) on employee behavioral responses. Social Indicators Research, 22, 31- 47.

Fernández Losa, N. (2002). El desarrollo profesional de los trabajadores como ventaja competitiva de las empresas. Cuadernos de Gestión, 2(1), 65-90.

Fonseca Lupiac, K.J. (2007). Los recursos tangibles, intangibles y las capacidades como factores explicativos de la competitividad empresarial de las mipymes del sector industrial en Nicaragua. (Tesis inédita de doctorado). Universidad Católica de Honduras.

French, W. (1996). Desarrollo Organizacional, Editorial Prentice Hal.

Gamero, G. (2003). Análisis económico de la satisfacción laboral. (Tesis inédita de doctorado). Facultad de ciencias económicas y empresariales, departamento de economía aplicada (estadística y econometría). Universidad de Málaga.

García Meier, E. (2014). Percepción de Calidad de Vida Laboral del personal operativo y administrativo en empresas de consumo masivo. (Tesis inédita de licenciatura). Universidad Rafael Urdaneta.

Gibson, I.D. (1996). Las Organizaciones, Editorial McGraw Hill, Madrid. 
González, L. J. (2009). Tendencias en la calidad de vida en países con dependencia mineral. Revista de Ciencias Sociales, 15(2). Maracaibo, Venezuela, 272-283.

Harter, J.K., Schmidt, F.L., y Hayes, T.L. (2002). Business unit-level relationship between employee satisfaction, employee engagement, and business outcomes: A meta-analysis, Fournal of Applied Psychology, 87(2), 268-279.

Hernández, R., Fernandez, G. y Baptista, P. (2003). Metodología de la investigación. Editorial Mac GrawHill. Chile.

Huerta, Soler y Duran (2003). Evaluación de la Calidad de Vida Laboral del personal administrativo de IPPLUZ. Unversidad Rafael Belloso Chacín. Venezuela.

Lau, R. y May, B. (1998). A Win- Win Paradigm for Quality of Worklife and Bussiness Performance. Human Resource Developpment Quarterly, 9(3), 211- 227.

Prahalad, G. y Hamel, G. (1990). The core competence of the corporation. Harvard Business Review, mayo-junio, .79-91.

Rubio Bañón, A. y Aragón Sánchez, A. (2006) Recursos estratégicos en las pymes. Revista Europea de Dirección y Economía de la Empresa, 17(1). 103-126.

Sánchez Ortiz, D.L. (2013). Influencia de la Calidad de Vida Laboral en los Individuos y las Organizaciones. Universidad de La Sabana, Facultad de Psicología. Disponible en: https:// intellectum.unisabana.edu.co/bitstream/handle/10818/9788/Dayana\%20Lorena\%20 S\%C3\%Alnchez $\% 20$ Ortiz $\% 20 \% 28$ TESIS\%29.pdf? sequence=1\&isAllowed $=\mathrm{y}$

Secretaria de Turismo. (2017). Empleo Turístico, Datatur. Disponible en: http://www.datatur. sectur.gob.mx/SitePages/ResultadosITET.aspx

Solleiro, J. y Castañón, R. (2005). Competitividad y sistemas de Innovación: Los retos para la inserción de México en el Contexto Global. Revista Iberoamericana, 5, p.15.

Ukko, J., Tenhunen, J. y Rantanen, H. (2008). The Impacts of Performance Measurement on the Quality of Working Life. Business Performance Management, 10(1), 86-98.

Wernerfelt, B. (1991). A Resource-Based View of The Firm. Strategic Management Journal, 5, 171-180. 
Zohurul, I. y Siengthai, S. (2009). Quality of Work Life and Organizational Performance: Empirical Evidence from Dhaka Export Processing Zone. This paper is prepared for the ILO Conference on 'Regulating for Decent Work, to be held at the International Labour Office, Geneva. 